\title{
Cerebral Amyloid Angiopathy with Symptomatic or Occult Subarachnoid Haemorrhage
}

\author{
K. Karabatsou ${ }^{a} \quad$ B.R.F. Lecky ${ }^{b} \quad$ N.G. Rainov ${ }^{a} \quad$ J.C. Broome ${ }^{c}$ R.P. White ${ }^{b}$ \\ Departments of ${ }^{a}$ Neurosurgery, ${ }^{b}$ Neurology and ${ }^{c}$ Neuropathology, Walton Centre for Neurology and \\ Neurosurgery, Liverpool, UK
}

\section{Dear Sir,}

Cerebral amyloid angiopathy (CAA) is a degenerative vasculopathy due to deposition of amyloid material within cortical and meningeal arterioles. In the common sporadic form, the predominant amyloid material is amyloid-beta $(A \beta)$. CAA manifests a spectrum from symptom-free disease to haemorrhage, ischaemic events and progressive dementia $[1,2]$. It is seldom described as a cause of subarachnoid haemorrhage (SAH) [3, 4]. We report a series of three patients with clinically manifest or occult SAH in which histopathology, clinical progression and neuro-radiological findings are consistent with CAA (Boston criteria [5]). Imaging from the cases illustrates a gradient echo MRI (GE-MRI) sign seldom discussed in the literature of CAA [3]: focal cortical superficial siderosis.

\section{Case Reports}

Case 1

A 58-year-old Caucasian woman presented with a 6-week history of headaches. She reported transient right-sided weakness associated with speech difficulty for minutes. She was a non-smoker without vascular risk factors. Following admission she developed right-sided focal motor seizures, controlled with phenytoin $300 \mathrm{mg}$ o.i.d. She had evidence of nominal aphasia.

CT of the brain demonstrated bilateral parietal SAH and subcortical periventricular low attenuation. CSF was positive for bilirubin on spectrophotometry. An MRI scan confirmed SAH and ischaemic leuko- araiosis (fig. 1). Routine blood tests including markers for vasculitis and coagulopathy were negative. A drug abuse screen was negative. A four-vessel digital subtraction angiogram was normal.

A brain and dura biopsy of the right frontal lobe was performed to exclude primary CNS vasculitis. The biopsy showed hyaline thickening of the walls of medium- and small-sized arteries and arterioles in the meninges, with apple-green birefringence on Congo red staining (fig. 2).

The patient was diagnosed with clinically probable CAA with supporting pathology [5]. She was readmitted 8 weeks later with a recurrent acute $\mathrm{SAH}$ as evidenced by acute headache and subarachnoid blood on CT over the left parietal area. This was managed conservatively and on review 12 months later she remains well.

\section{Case 2}

A hypertensive 72-year-old Caucasian man, he presented with transient episodes of left-sided weakness and paraesthesia. These were considered crescendo TIAs and he received intravenous heparin and carotid imaging. His symptoms settled and carotid ultrasound was normal. CT brain showed subcortical leukoaraiosis only. Echocardiography was unremarkable. He was discharged on single agent anti-platelet therapy (aspirin $75 \mathrm{mg}$ o.i.d.) but readmitted with recurrent attacks. A diagnosis of capsular warning syndrome

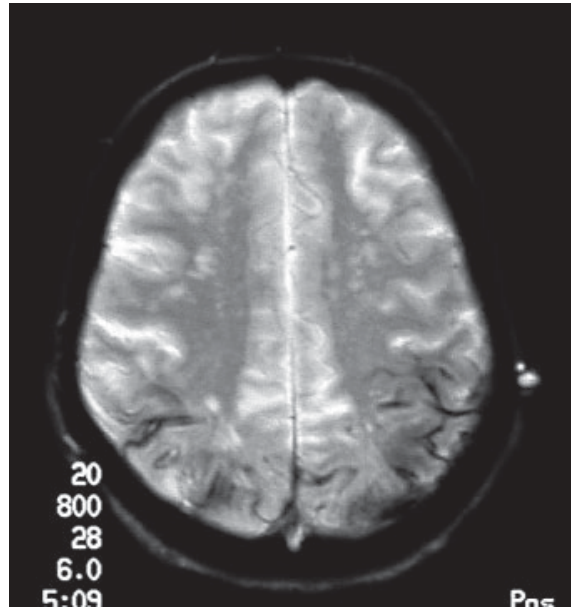

Fig. 1. $T_{2}$ MRI of case 1 illustrating acute SAH over bi-parietal regions and subcortical leukoaraiosis.

was made. He was discharged on dual antiplatelet therapy (aspirin $75 \mathrm{mg}$ o.i.d. and clopidogrel 75 mg o.i.d.), but attacks continued.

He presented 4 months later with confusion and bi-frontal headache. He developed generalized seizures controlled with phenytoin (loading dose, followed by $300 \mathrm{mg}$ o.i.d.).

CT brain showed left parietal and right frontal acute subcortical ICH. There was no evidence of acute SAH. MRI venography and intracranial MRA were normal.

\section{KARGER}

Fax +4161306 1234 E-Mail karger@karger.ch www.karger.com
(C) 2007 S. Karger AG, Base 0014-3022/07/0572-0103\$23.50/0

Accessible online at: www.karger.com/ene
Dr. R.P. White

Walton Centre for Neurology and Neurosurgery

Lower Lane, Fazakerley, Liverpool L9 7LJ (UK)

Tel. +44151529 6260, Fax +441515295512

E-Mail richard.white@thewaltoncentre.nhs.uk 
Fig. 2. Congo red staining of leptomeningeal vessel demonstrating apple-green birefringence under polarized light.

Fig. 3. GE-MRI for patient 2, demonstrating acute subcortical haematoma involving right frontal and left parietal regions, and superficial siderosis of the right fronto-parietal region.

Fig. 4. GE-MRI for patient 3, demonstrating superficial siderosis of the right sensory cortex and a clinically silent subcortical haematoma not seen on standard $\mathrm{T}_{2}$ sequences.

GE-MRI showed superficial siderosis of the right parietal cortex consistent with previous clinically occult $\mathrm{SAH}$, in addition to his bilateral intracerebral bleeds (fig. 3).

A diagnosis of clinically probable CAA [5] was made and his anti-platelet drugs discontinued. He made a good recovery and was stable for 12 months, at which

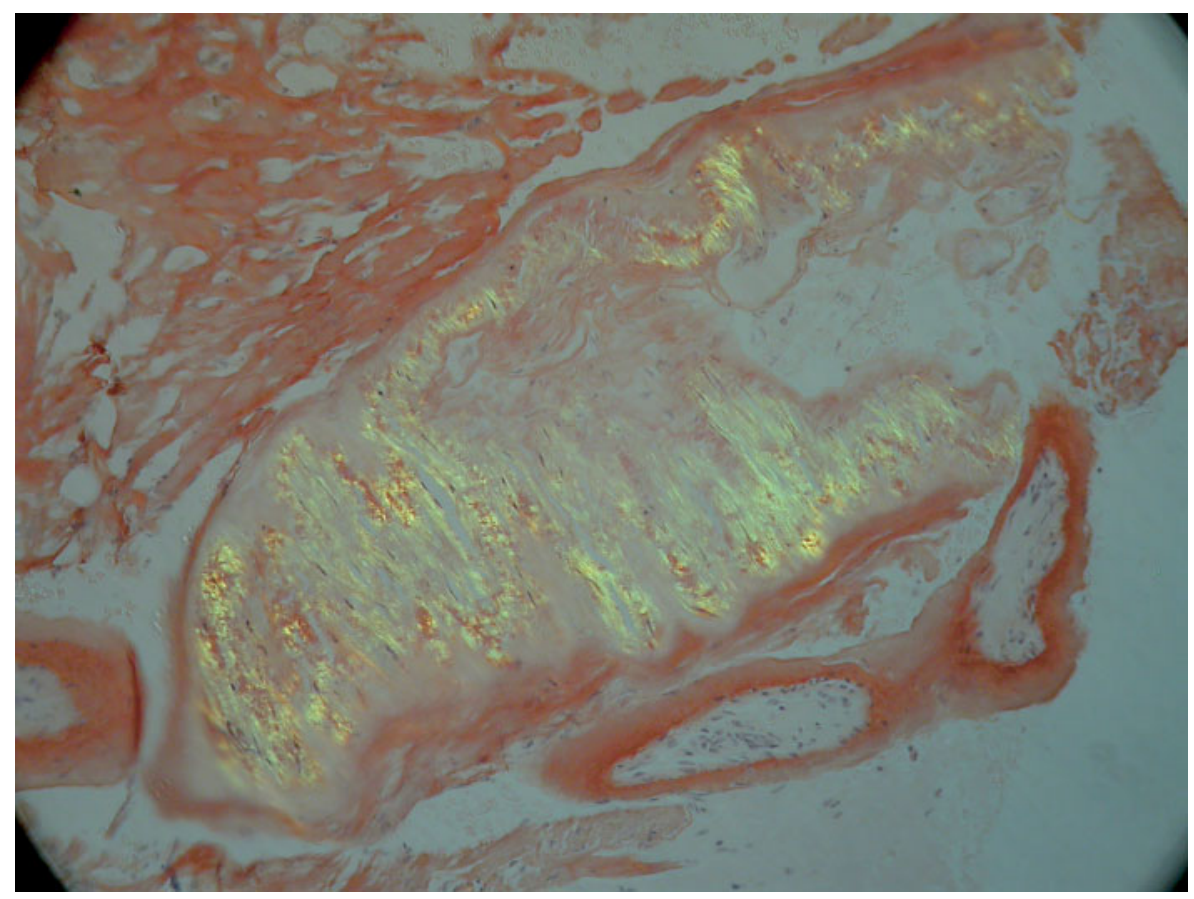

3

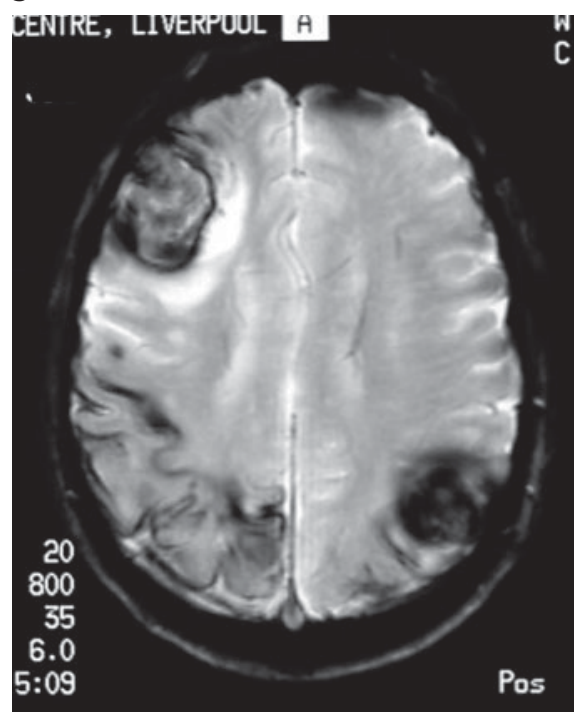

point he developed right arm sensory-motor attacks, which settled on phenytoin $300 \mathrm{mg}$ o.i.d. Over a 3-year follow-up period there has been no cognitive decline.

\section{Case 3}

A 75-year-old Caucasian woman without vascular risk factors presented with recurrent left facio-brachial numbness. Over
4

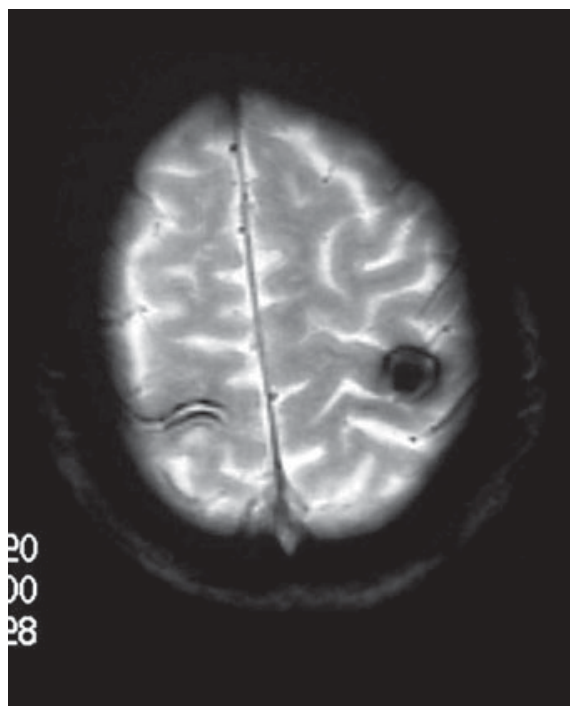

a 5-month period she had been experiencing attacks of numbness and paraesthesia over the upper lip followed by tingling of the left thumb progressing to involve all fingers. Episodes lasted for a 2-3 min and there were no associated motor or cognitive features. Attacks were irregular but often clustered with 10 or more occurring over a period of $24-48 \mathrm{~h}$. 
Clinical examination was normal. Electrocardiography and sleep-deprived electroencephalography were normal. Carotid Doppler ultrasound was normal. Routine laboratory parameters were within normal limits.

Focal cortical siderosis of the right sensory cortex on GE-MRI suggested occult SAH (fig. 4). The subcortical deposition of haemosiderin in the left parietal region (not evident on $\mathrm{T}_{2}$ ) suggested silent old primary intracerebral haemorrhage. Intracranial MRA was normal.

A diagnosis of clinically probable CAA was made [5]. The sensory attacks were interpreted as seizures and resolved on carbamazepine (300 mg b.i.d). She remains well at annual review.

\section{Discussion}

We describe primary SAH as a first presentation of probable CAA with supporting pathology [5]. We have also described two cases with clinically probable CAA [5] who presented with recurrent sensory or sensorimotor attacks and radiological evidence of focal cortical superficial siderosis. The authors consider this to be evidence of occult SAH.

CAA has received little attention in the literature as a cause of primary $\mathrm{SAH}[3,4]$. More typically in CAA, SAH is assumed to be secondary to extension from $\mathrm{ICH}$. The landmark feature of CAA is media degeneration. The development of haemorrhage occurs in stages. Accumulation of amyloid leads to loss of smooth muscle cells and entry of plasma components. With deposition growth, vessel wall is replaced, eventually rupturing to cause haemorrhage [ 9 , 10]. Amyloid infiltration of cerebral vessels may cause narrowing of the vascular lumen, fibrinoid necrosis and occlusion factors that predispose to transient cerebral ischaemia and stroke [7, 10, 11]. A large necropsy series reported CAA to be rarely associated with SAH although it appears to be the most frequent cause of ICH with secondary SAH in the elderly [6]. This study and others [2,7], suggest the scarcity of CAA-related SAH reflects differential amyloid deposition between cortical and leptomeningial vessels.

Recent evidence suggests the initial haemorrhage occurs in the subarachnoid space. Takeda et al. [8] examined six au- topsy cases of subcortical haematoma caused by CAA in order to identify the primary sites of the haemorrhage. They concluded that the primary haemorrhage occurred in the subarachnoid space of the cerebral sulci, due to rupture of meningeal arteries. The cortical area around the haematoma infarcts and further haemorrhagic extensions penetrate the subcortical parenchyma, causing haematoma enlargement. Because of the small size of this study caution is required in extrapolating these observations in general, which have not yet been confirmed by other workers.

CAA diagnosis can be made from haematoma evacuation tissue or cortical biopsy, but the sensitivity and specificity depend on age, site and type of biopsy [12, 13]. Frequently the diagnosis cannot be confirmed until autopsy [13]. Without an effective treatment for CAA clinicians are reluctant to biopsy and diagnosis is clinical - recurrent sub-subcortical haemorrhage or coincidental bilateral haemorrhages, in the absence of other causes. Diagnostic criteria have been proposed and validated [5]. GE-MRI provides a powerful tool since it is sensitive to the presence of haemosiderin from old haemorrhages, not otherwise apparent $[12,14]$. We suggest that CAA may be under-appreciated as a cause of SAH and cortical superficial siderosis is diagnostically supportive of $\mathrm{SAH}$ in CAA. We suggest that this provides one explanation for the recurrent sensori-motor symptoms described in CAA [14], although it is unclear whether they are of seizure or ischaemic semiology. Our cases response to anti-convulsants suggests it is the former.

Recurrent sensorimotor symptoms provide a clinical 'trap' if crescendo TIAs are considered and treated with escalating doses of anti-thrombotic agents. GE-MRI may be warranted in such situations to prevent the inappropriate use of antithrombotic drugs in patients with CAA.

\section{References}

1 Greenberg S: Cerebral amyloid angiopathy prospects for clinical diagnosis and treatment. Neurology 1998;51:690-694.

2 Gilbert JJ, Vinters HV: Cerebral amyloid angiopathy: incidence and complications in the aging brain.1. Cerebral hemorrhage. Stroke 1983;14:915-923.
3 Jin K, Sato S, Takahashi T, Nakazaki N, Date Y, Nakazato M, Tominaga T, Itoyama Y, Ikeda S: Familial leptomeningeal amyloidosis with a transthyretin variant Asp18Gly representing repeated subarachnoid haemorrhages with superficial siderosis. J Neurol Neurosurg Psychiatry 2004;75:1463-1466.

4 Ohsima T, Endo T, Nukui H, Ikeda S, Allsop $\mathrm{D}$, Onaya T: Cerebral amyloid angiopathy as a cause of subarachnoid hemorrhage. Stroke 1990;21:480-483.

5 Knudsen KA, Rosand J, Karluk D, Greenberg S: Clinical diagnosis of cerebral amyloid angiopathy: validation of the Boston Criteria. Neurology 2001;56:537-539.

6 Yamada M, Itoh Y, Otomo E, Miyatake T: Subarachnoid hemorrhage in the elderly: a necropsy study of the association with cerebral amyloid angiopathy. J Neurol Neurosurg Psychiatry 1993;56:543-547.

7 Mandybur T: Cerebral amyloid angiopathy: the vascular pathology and complications. J Neuropathol Exp Neurol 1986;45:79-90.

8 Takeda S, Yamazaki K, Miyakawa T, Onda K, Hinokuma K, Ikuta F, Arai H: Subcortical hematoma caused by cerebral amyloid angiopathy: does the first evidence of hemorrhage occur in the subarachnoid space? Neuropathology 2003;23:254-261.

9 Alonzo N, Hyman B, Rebeck W, Greenberg $S$ : Progression of cerebral amyloid angiopathy: accumulation of amyloid- $\beta 40$ in affected vessels. J Neuropathol Exp Neurol 1998; 57:4:353-359.

10 Zhang W, Lempessi H, Olsson Y: Amyloid angiopathy of the human brain: immunohistochemical studies using markers for components of extracellular matrix, smooth muscle actin and endothelial cells. Acta Neuropathol 1998;96:558-563.

11 Smith D, Hitchcock M, Philpot P: Cerebral amyloid angiopathy presenting as transient ischemic attacks. J Neurosurg 1985;63:963964.

12 Hendricks H, Franke C, Theunissen P: Cerebral amyloid angiopathy: diagnosis by MRI and brain biopsy. Neurology 1990;40:13081310.

13 Greenberg S, Vonsattel J: Diagnosis of cerebral amyloid angiopathy. Sensitivity and specificity of cortical biopsy. Stroke 1997;28: 1418-1422.

14 Roch JA, Nighoghossian N, Hermier M, Cakmak S, Picot M, Honnorat J, Derex L, Trouillas P: Transient neurologic symptoms related to cerebral amyloid angiopathy: usefulness of $\mathrm{T}_{2}{ }^{*}$-weighted imaging. Cerebrovasc Dis 2005;20:412-414. 